\title{
The Earliest Fleshy Cone of Ephedra from the Early Cretaceous Yixian Formation of Northeast China
}

\author{
Yong Yang, Qi Wang*
}

State Key Laboratory of Systematic and Evolutionary Botany, Institute of Botany, Chinese Academy of Sciences, Beijing, P. R. China

\begin{abstract}
Bracts of female cones of extant gymnosperm Ephedra (Joint fir) are either colorful and fleshy (section Ephedra), or drywinged and membranous (section Alatae), or dry and coriaceous (section Asarca), which have played a crucial role in longdistance seed dispersal that is responsible for a wide distribution of the genus in semiarid and arid areas of Eurasia, North Africa, North America, and South America. Recent molecular systematic studies on Ephedra have suggested that the fleshy bracts in character evolution may be plesiomorphic relative to the dry, membranous and coriaceous bracts. However, little is known about when the fleshy bracts of Ephedra have made their debut in the geological past. Herein, we describe a novel, fleshy bract-bearing female cone macrofossil from the Early Cretaceous (ca. 120-125 Ma) Yixian Formation in Liaoning, northeastern China. This cone bears three ellipsoid seeds subtended by only one whorl of fleshy bracts. Each seed has a thin outer envelope and an inner integument that extends upward and passes through the opening of the outer envelope, forming a thin and straight micropylar tube. Such a syndrome shows the closest similarity to an extant triovulate species Ephedra intermedia in the section Ephedra, but the latter bears a whorl of terminal fertile bracts and more than one whorl of inferior sterile bracts, and a thick outer envelope. Hence, we establish a new fossil species Ephedra carnosa. Our discovery provides the first direct macrofossil evidence for the previous molecular systematics of Ephedra, implying that the origin of fleshy bracts in Ephedra should not have been later than that of the membranous and coriaceous bracts by at least the Early Cretaceous.
\end{abstract}

Citation: Yang Y, Wang Q (2013) The Earliest Fleshy Cone of Ephedra from the Early Cretaceous Yixian Formation of Northeast China. PLoS ONE 8(1): e53652. doi:10.1371/journal.pone.0053652

Editor: Lee A. Newsom, The Pennsylvania State University, United States of America

Received May 21, 2012; Accepted December 3, 2012; Published January 14, 2013

Copyright: ๑ 2013 Yang, Wang. This is an open-access article distributed under the terms of the Creative Commons Attribution License, which permits unrestricted use, distribution, and reproduction in any medium, provided the original author and source are credited.

Funding: The study was supported by the National Natural Science Foundation of China (no. 30970177, 30600035, 40830209, 40972015), the State Key Laboratory of Systematic and Evolutionary Botany, Institute of Botany, Chinese Academy of Sciences (no. 56176G1044) to WQ and YY. The funders had no role in study design, data collection and analysis, decision to publish, or preparation of the manuscript.

Competing Interests: The authors have declared that no competing interests exist.

*E-mail: happyking@ibcas.ac.cn

\section{Introduction}

The gymnospermous genus Ephedra L. (Joint fir) contains about 50 living species, native to semiarid and arid areas of Asia, Europe, North Africa, North America, and South America ([1-7]; Fig. 1). This genus has three types of female cones upon which a once widely accepted sectional classification is based [8], i.e., Sect. Alatae Stapf bears free, dry, winged, and membranous bracts (Fig. 2A), Sect. Asarca Stapf has free, dry, but coriaceous bracts (Fig. 2B), while Sect. Ephedra possesses thickened, colorful, and fleshy bracts (Fig. 2C). Recent molecular phylogenetic studies have suggested that the three morphological sections are not natural and none of them is monophyletic, but the earliest diverged branch within the genus comprises species with fleshy cones from the Mediterranean region [9-12]. Meanwhile, Sect. Alatae and Sect. Asarca are nested within Sect. Ephedra, implying that the fleshy cone is plesiomorphic in Ephedra. However, when the first fleshy cone occurred in the fossil record remains unknown. Ephedra macrofossils (especially female cones) will provide an historical perspective for the early evolution, taxonomy, and biogeography of the genus.

Early Ephedra might have transformed bracts of female cones into fleshiness and vivid color to assist seed dispersal [13-15], resulting in a wide intercontinental distribution of the genus. However, little is known about when such characteristic fleshy cones occurred in Ephedra. According to molecular clock data, modern Ephedra was estimated to have a minimum age 8-32 Ma (i.e., an Oligocene origin) $[11,16]$. However, it lags behind the first occurrence of Early Cretaceous meso- and macrofossils bearing clear morphology that can be reliably circumscribed within Ephedra, e.g., E. archaeorhytidosperma Yang et al. [17], E. portugallica Rydin et al., E. drewriensis Rydin et al. [18], and E. hongtaoi Wang et Zheng [19]. Therefore, pruning of early stem groups and extinctions of ancient lineages may have played an important role in the early evolution of Ephedra [11,12,20]. It is expected that the putatively plesiomorphic character bearing fleshy cones in Ephedra would have made their debut earlier than Oligocene.

In recent decades, numerous Ephedra and Ephedra-like meso- and macrofossils have been reported from the Early Cretaceous of South Europe, Northeast China, Mongolia, North America, and South America [17-19,21-23]. Seed mesofossils with in situ pollen were reported from the Early Cretaceous of South Europe (Portugal) and North America [18,24]. Macrofossils of reproductive shoots or female cones were found in the Early Cretaceous of South America [25,26], and Mongolia [27,28] and adjacent Northeast China [17,19,21-23,29,30]. Early Cretaceous strata of Northeast China contain a number of well-preserved ephedroid macrofossils that may shed light on the early evolution of Ephedra. They show high reproductive diversity but similar vegetative morphology, e.g., dichasial branching pattern (sometimes branches being clustered due to highly shortened internodes), long and 


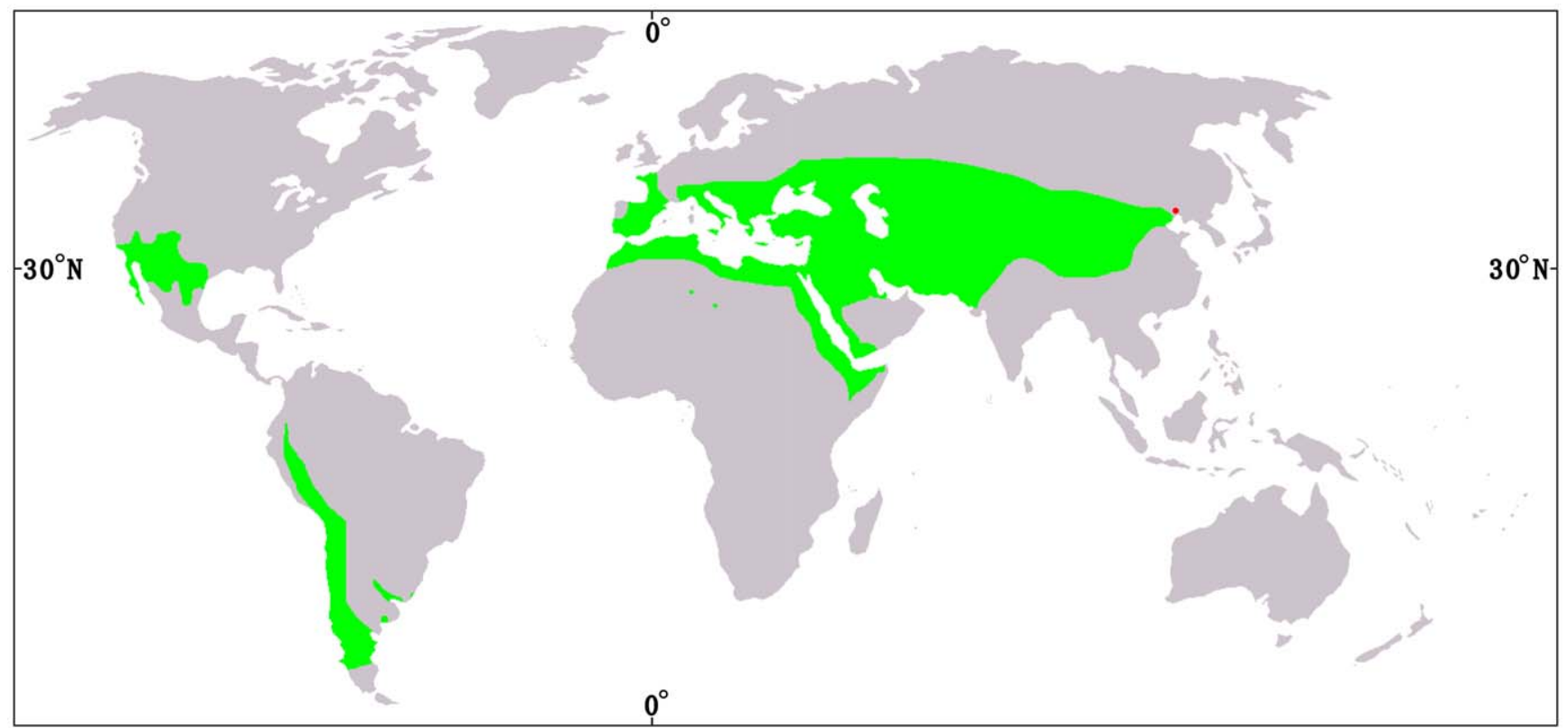

Figure 1. Distribution of extant Ephedra (green regions) after [5] (red dot showing the present fossil locality). doi:10.1371/journal.pone.0053652.g001

linear leaves usually with two parallel veins, and internodes with longitudinal fine striations, which can be divided into three groups. The first group bears female cones with multiple whorls of fertile bracts, e.g. Liaoxia Cao et $\mathrm{Wu}$ (= Ephedrites Göppert et Berendt) $[21,31]$; the second group bears female cones with only one whorl of fertile bracts, e.g., Ephedra hongtaoi and E. archaeorhytidosperma $[17,19]$ and several species ascribed to Gurvenella Krassilov (= Chaoyangia Duan) bearing trichotomous complex surrounding female cones [27,32,33]; the third group is Siphonospermum Rydin et Friis which has female cones without supporting bracts [22]. So far, all the previous studies have not provided any fossil evidence for the origin of fleshy female cones of Ephedra.

In this paper, we aim to describe a new, freshy cone-bearing fossil species, Ephedra carnosa Yang et Wang sp. nov., from the Early Cretaceous of Liaoning Province, Northeast China. Our discovery provides the first direct macrofossil evidence for the previous molecular systematics of Ephedra, implying that the origin of fleshy bracts in Sect. Ephedra should not have been later than that of the membranous and coriaceous bracts in Sect. Alatae and Sect. Asarca by at least the Early Cretaceous.

\section{Materials and Methods}

The macrofossils used in this study were collected from the Yixian Formation at Huangbanjigou Village of Shangyuan Town, Beipiao City, Liaoning Province, Northeast China [Fig. 3]. The Yixian Formation is widely distributed in West Liaoning [33], and its geological age is the early Aptian - earliest late Aptian of the Early Cretaceous, which can be correlated by radiometric dating to about 120-125 Ma [34-39]. Previously, this formation has
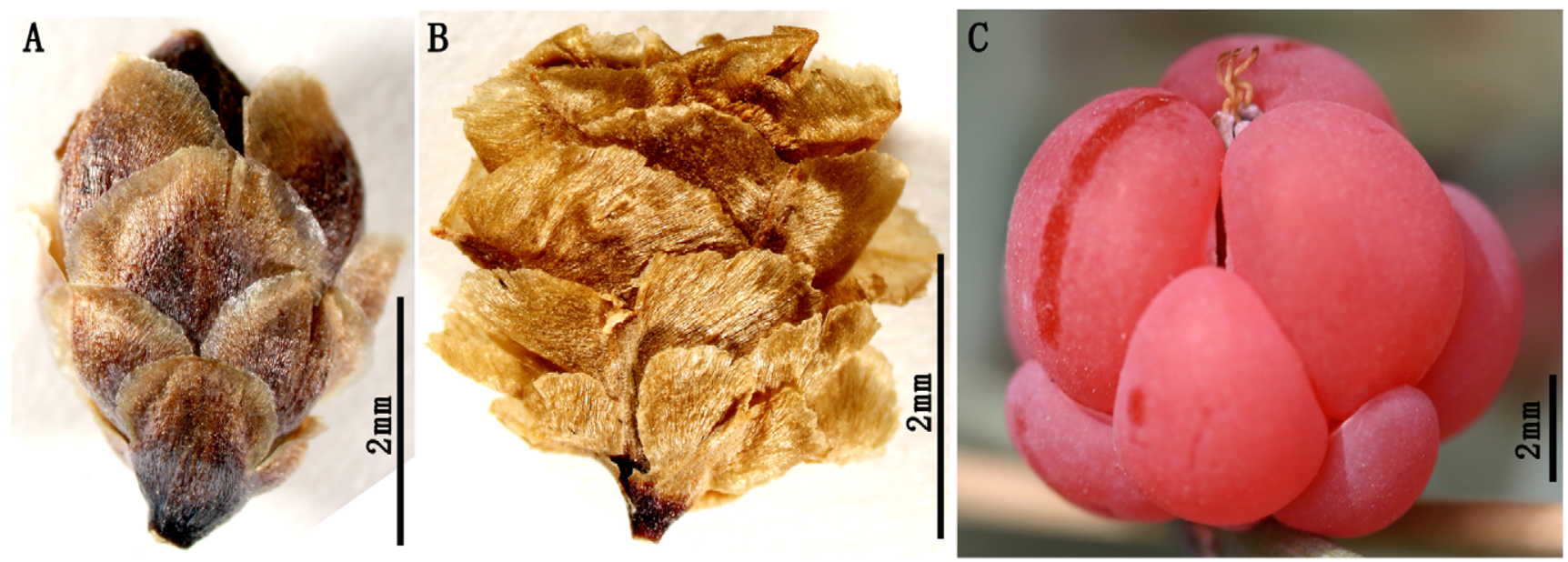

Figure 2. Representative female cones of three sections in Ephedra. A. A membranous female cone of E. californica Watson in Sect. Alatae Stapf. B. A coriaceous female cone of E. strobilacea Bunge in Sect. Asarca Stapf. C. A fleshy female cone of E. intermedia Schrenk et Mey. in Sect. Ephedra.

doi:10.1371/journal.pone.0053652.g002 


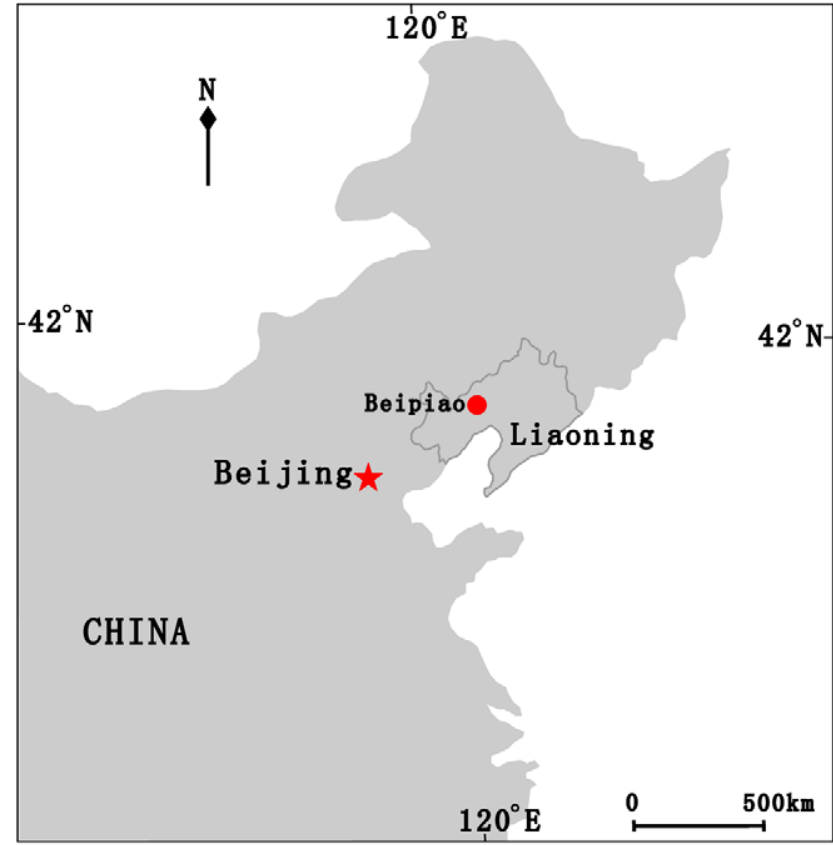

Figure 3. Fossil locality showing Beipiao (red dot) of Liaoning Province, Northeast China after [40]. doi:10.1371/journal.pone.0053652.g003

yielded a plethora of extraordinarily well-preserved freshwater and terrestrial fossils, especially including early angiosperms (e.g., Archaefructus liaoningensis Sun et al.), feathered theropod dinosaurs, early seed-eating birds, and primitive mammals [35-37,39-41].

The macrofossils are preserved as impressions lacking cuticle and include a part and a counterpart from a gathering slab of finely laminated light grey to yellowish siltstone. Figures 1, 3 presented here were redrawn from the base maps, respectively [5,40]. Voucher specimens (WH Lipsky 4181 and L Benson 15280) of Ephedra californica Watson and E. strobilacea Bunge were examined at the China National Herbarium (PE), including two female cones used in Figure 2A-B. The female cone of Ephedra intermedia Schrenk et Mey. presented in Figure 2C was photographed by the senior author at Mt Renshoushan of Gansu Province, Northwest China. Macrofossils were photographed with digital cameras (Nikon D700 and Panasonic DMC-FZ30) and under a microscope (Nikon Eclipse E600). Comparisons were made in Table 1 with some coeval, similar Ephedra and ephedroid meso- and macrofossils. All figures were arranged by Adobe Photoshop 6.0 and CorelDraw 10.0 programs. Terminology on the female cones of Ephedra [6,42-44] used in the specimen descriptions was adopted. The specimens (including two gatherings PE 20120319A and 20120319B, 2012071006) are deposited at the China National Herbarium (PE), Institute of Botany, Chinese Academy of Sciences, Beijing, P. R. China.

\section{Nomenclature}

The electronic version of this article in Portable Document Format (PDF) in a work with an ISSN or ISBN will represent a published work according to the International Code of Nomenclature for algae, fungi, and plants, and hence the new names contained in the electronic publication of a PLOS ONE article are effectively published under that Code from the electronic edition alone, so there is no longer any need to provide printed copies. The online version of this work is archived and available from the following digital repositories: PubMed Central, LOCKSS, BioOne.

\section{Results}

The three extant gymnospermous genera, Ephedra L., Gnetum L., and Welwitschia Hooker, have been widely treated as a natural taxon, either a class Gnetopsida or an order Gnetales, with their own monogeneric families $[3,6,7,45,46]$. Therefore, our new fossil species of Ephedra is classified as follows:

Gnetopsida Eichler ex Kirpotenko, 1884

Gnetales Luerss., 1879

Ephedraceae Dumort., 1829

Ephedra L., 1753

Ephedra carnosa Yang et Wang, sp. nov. (Fig. 4)

\section{Diagnosis}

Triovulate cones have but only one whorl of bracts. Each bract is apparently thickened and spreading and subtends a seed. The bract bears two veins sub-parallel in the middle-upper part and divergent toward the basal part. Seeds are ellipsoid. The outer envelope is thin. The inner integument is fused with the nucellus, but only its apical part extends upward and passes through the outer envelope opening, forming a slim and straight micropylar tube.

\section{Description}

The fossils presented here are triovulate cones, having but only one whorl of bracts [Fig. 4A-D, H-I]. A remnant receptacle is preserved at the bottom of the cone. The receptacle is $1.3 \mathrm{~mm}$ long and $1.3 \mathrm{~mm}$ wide at the base, and thickened acropetally up to $2.4 \mathrm{~mm}$ wide. The bracts are apparently thickened, spreading, triangular, and tapered [Fig. 4C-D, F-G], ca. 5-6 mm long and $3 \mathrm{~mm}$ wide at the base while $2 \mathrm{~mm}$ wide at the apex. The bract margin is not clearly defined. Each bract has two veins sub-parallel in the middle-upper part and divergent toward the basal part [Fig. 4F-G]. The interval between the two veins is wider at the base (up to $620 \mu \mathrm{m}$ ) and becomes narrower (ca. $207 \mu \mathrm{m}$ ) in the middle part, and then the two veins are parallel (ca. $138 \mu \mathrm{m})$ to each other towards the apex. Each bract subtends a seed. Seeds are ellipsoid, flat at the ventral side and arched at the dorsal side, about $1.5 \mathrm{~mm}$ wide and $2.6-3 \mathrm{~mm}$ long. The outer envelope is thin. The inner integument appears to be fused with the nucellus, but only its apical part extends upward and passes through the opening of the outer envelope to form a micropylar tube [Fig. 4E]. The micropylar tube is slim and straight, the exposed part is approximately $1.3 \mathrm{~mm}$ long.

\section{Etymology}

The specific epithet is derived from Latin "carnosus", denoting the apparently thickened (thereby fleshy) bracts of female cones.

\section{Holotype}

(Designated here) - PE 20120319A, B (part and counterpart), deposited at the China National Herbarium (PE), Institute of Botany, Chinese Academy of Sciences, Beijing, P. R. China.

\section{Paratype}

PE 2012071006, deposited at the China National Herbarium (PE), Institute of Botany, Chinese Academy of Sciences, Beijing, P. R. China. 
Table 1. Key to extant and fossil species in Ephedra and other ephedroids.

\begin{tabular}{|c|c|}
\hline \multicolumn{2}{|l|}{ 1. Female cones lacking supporting bracts ----_espermum [22] } \\
\hline \multicolumn{2}{|l|}{ 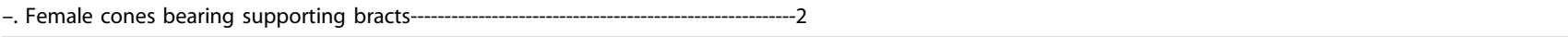 } \\
\hline \multicolumn{2}{|l|}{ 2. Female cones bearing multiple pairs/whorls of fertile bracts---_-_-_--_iaoxia [21] } \\
\hline \multicolumn{2}{|l|}{-. Female cones bearing 1 pair/whorl of fertile bracts-- } \\
\hline \multicolumn{2}{|l|}{ 3. Female cones surrounded by furcated appendages--------------------Gurvanella [27] } \\
\hline \multicolumn{2}{|l|}{-. Female cones lacking furcated appendages-- } \\
\hline \multicolumn{2}{|l|}{ 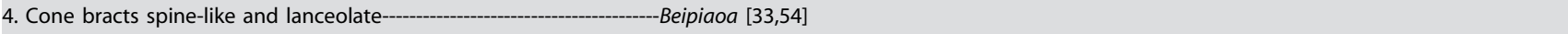 } \\
\hline \multicolumn{2}{|l|}{-. Cone bracts obtuse or acute when present-- } \\
\hline \multicolumn{2}{|c|}{$\begin{array}{l}\text { 5. Female cones bearing only a pair/whorl of terminal, fertile bracts and 1-multiple pair/whorls of inferior sterile bracts-- } \\
\text { [44] }\end{array}$} \\
\hline \multicolumn{2}{|c|}{$\begin{array}{l}\text {-. Female cones having only a whorl of terminal, fertile bracts and lacking or having only a pair/whorl of inferior sterile bracts------------ fossil } \\
\text { Ephedra } 6\end{array}$} \\
\hline \multicolumn{2}{|c|}{$\begin{array}{l}\text { 6. Female cones having only a pair of inferior sterile bracts, and seed surface bearing transverse ridges--- } \\
\text { [17] }\end{array}$} \\
\hline \multicolumn{2}{|l|}{ 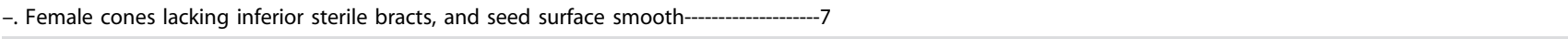 } \\
\hline \multicolumn{2}{|l|}{ 7. Biovulate, and micropylar tube less than $0.8 \mathrm{~mm}-$} \\
\hline -. Triovulate, and micropylar tube longer than $1 \mathrm{~mm}---------E . ~ c a r n o s a$ [this paper] & \\
\hline
\end{tabular}

\section{Locality}

Huangbanjigou Village of Shangyuan Town, Beipiao City, Liaoning Province, Northeast China.

\section{Stratigraphic horizon and age}

Yixian Formation, the early Aptian - earliest late Aptian of the Early Cretaceous.

\section{Comparisons}

The new fossil species Ephedra carnosa Yang et Wang is noticeably different from any other known extant and fossil species in Ephedra and other ephedroids [Table 1].

\section{Remarks}

Bracts of female cones of Ephedra are modified foliar organs in nature. In living Ephedra, cone bracts have three states according to their mature morphology, e.g., fleshy bracts, dry and coriaceous bracts, and dry and membranous bracts (see Introduction, Fig. 2AC). The thickness of median portion of cone bracts between the two parallel vascular bundles varies in the three sections of Ephedra, Sect. Ephedra (1.2-1.8 mm in E. intermedia), Sect. Asarca (ca. $80 \mu \mathrm{m}$ in E. californica), and Sect. Alatae (ca. $40 \mu \mathrm{m}$ in E. torreyana) (unpublished personal observations). This demonstrates that the fleshy cone bracts are markedly thicker than dry (either coriaceous or membranous) bracts in Ephedra. Similarly, the triovulate cones of our new macrofossil species Ephedra carnosa bear apparently thickened bracts without clearly defined margins, which are far more likely to be compared with those fleshy bracts in living Ephedra. In addition, two veins in each bract are sub-parallel in the middle-upper part (ca. 138-207 $\mu \mathrm{m}$ apart) but apparently divergent toward the basal part (up to $620 \mu \mathrm{m}$ apart), implying that the bract would be most possibly swollen in life. Hence, the cone bracts of macrofossils Ephedra carnosa presented here are ripe and fleshy.

The triovulate cones appear to have abscised from the reproductive shoots as a mature disseminule (or diaspore). Almost intact micropylar tubes in fossils imply that the cones have not been transported far from the parent plants before fossilization.
On the basis of its trimerous nature, three seeds of each cone must be subtended by three verticillate bracts, which are 120 degrees distant from each other. Two bracts are visible, so the third bract might have run into the embedding rock after the cone fell into soft sediments, fossilized and then opened along the cutting plane. Building on the above morphological description and taphonomic inference, we present here a schematic reconstruction of the triovulate cone and its seed [Fig. 5].

There is an associated axis in the specimens besides the female cone described above. The axis is straight, $1.3 \mathrm{~cm}$ long and $0.4 \mathrm{~mm}$ wide, with fine longitudinal striations (Fig. 4A-B). These features imply that the axis is likely to be the vegetative twigs of a same parent plant (or population) as the female cone. Due to lacking organic connection and other useful epidermal evidence between them, we do not include the axis into the description of this new species.

\section{Discussion}

\section{Characteristic thin outer envelope}

The outer envelope of the new macrofossil species Ephedra carnosa seems very thin and vulnerable, so it is easy to be transformed after compression. Also, the integument extended into a micropylar tube enclosed in the outer envelope is visible. In contrast, modern Ephedra with fleshy bracts usually bears a thicker and harder outer envelope and a very thin integument with only $1-2$-cell thick $[44,45]$. In addition, Sect. Alatae usually bears thin outer envelope and integument while Sect. Asarca and Sect. Ephedra usually bear a thickened outer envelope. As a result, our new fossil species is quite unique in that it bears fleshy bracts and a very thin outer envelope.

The outer envelope of modern Ephedra has three types of ornamentations, including smooth surface (e.g., E. intermedia, E. distachya L., E. aphylla Forssk., and E. tweediana Fisch. et Mey.), papillate type (e.g., E. major Host, and E. funerea Coville et Morton), and transverse lamellar sculpture (e.g., E. rhytidosperma Pachomova, E. trifurca Torr. $[6,47,48]$. The smooth and/or striated surfaces are very common in both extant and fossil species while the other two types are only restricted to a few species. The transverse lamellar 


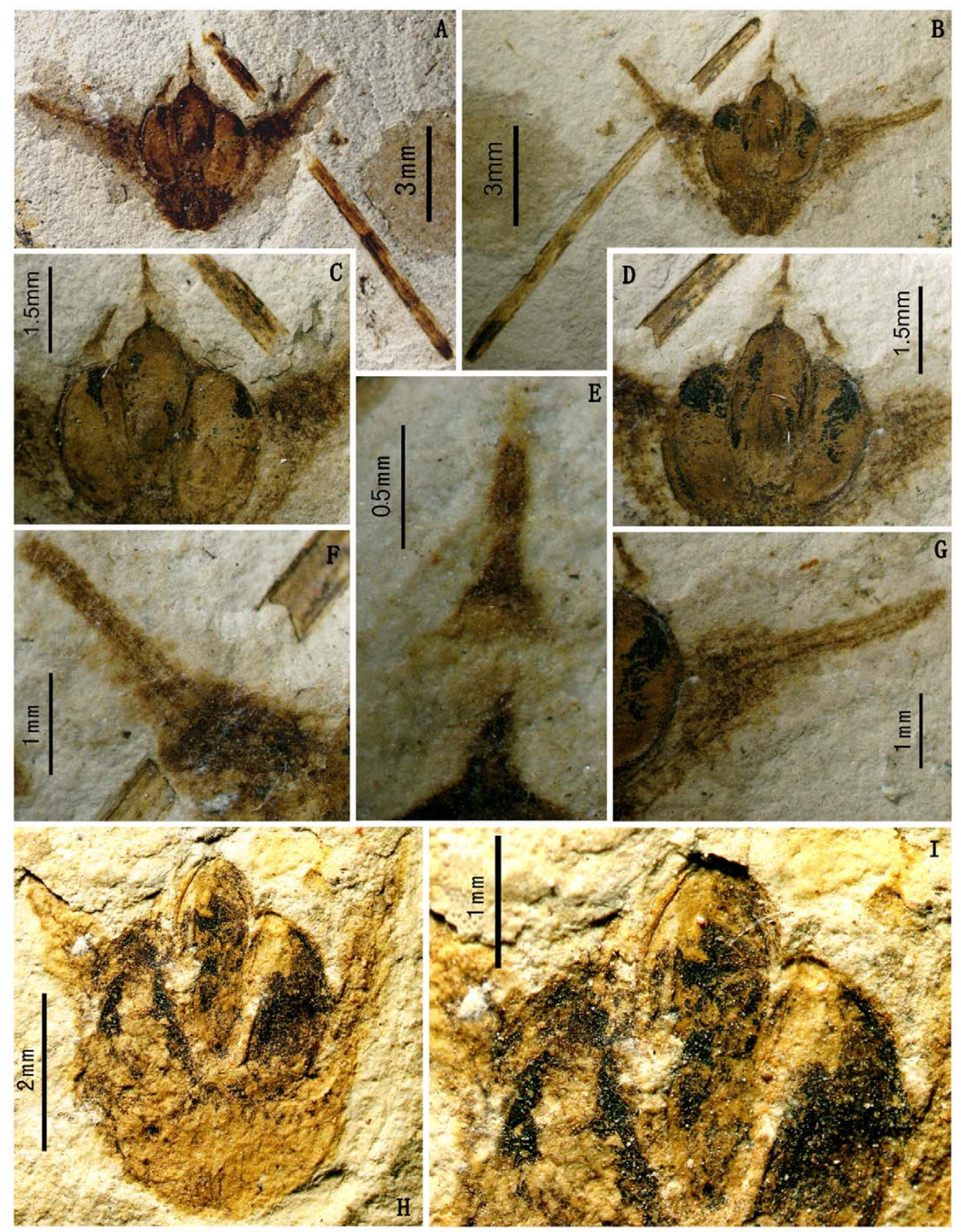

Figure 4. Fossil Ephedra carnosa Yang et Wang. A-B. A fleshy female cone and an associated axis. Holotype: PE 20120319A, B (part and counterpart). C-D. Enlargment of the female cone, showing morphology of three seeds. E. Close-up of the middle seed, showing a thin outer envelope and a straight micropylar tube. PE 20120319A. F-G. Close-up of the fleshy bract, bearing two veins sub-parallel in the middle-upper part and divergent toward the basal part. H. Another fleshy female cone. Paratype: PE 2012071006. I. Close-up of three seeds in Fig. H. doi:10.1371/journal.pone.0053652.g004

sculpture can also be found in fossil species from the Early Cretaceous [17,49-51] and might have multiple origins [52]. Our new fossil species Ephedra carnosa bears the outer envelope with the smooth type of seed surface. Surface sculpture of the outer envelope may be variable in the developmental sequence. In both $E$. equisetina Bunge and E. rhytidosperma, the outer envelope is generally smooth at the early stages of development while specialized surface ornamentations only occur in the late stages of development [6]. In modern Ephedra, fleshiness is correlated with maturity of female cones. As a result, we infer that our new fossil species has ripe reproductive units with smooth outer envelope. Such a thin outer envelope may have some physiological functions, e.g., regulating water loss.

Two alternative hypotheses may be used to explain the thin outer envelope of this new fossil Ephedra. One is that this new species represents the stem lineage of Ephedra and it is the 


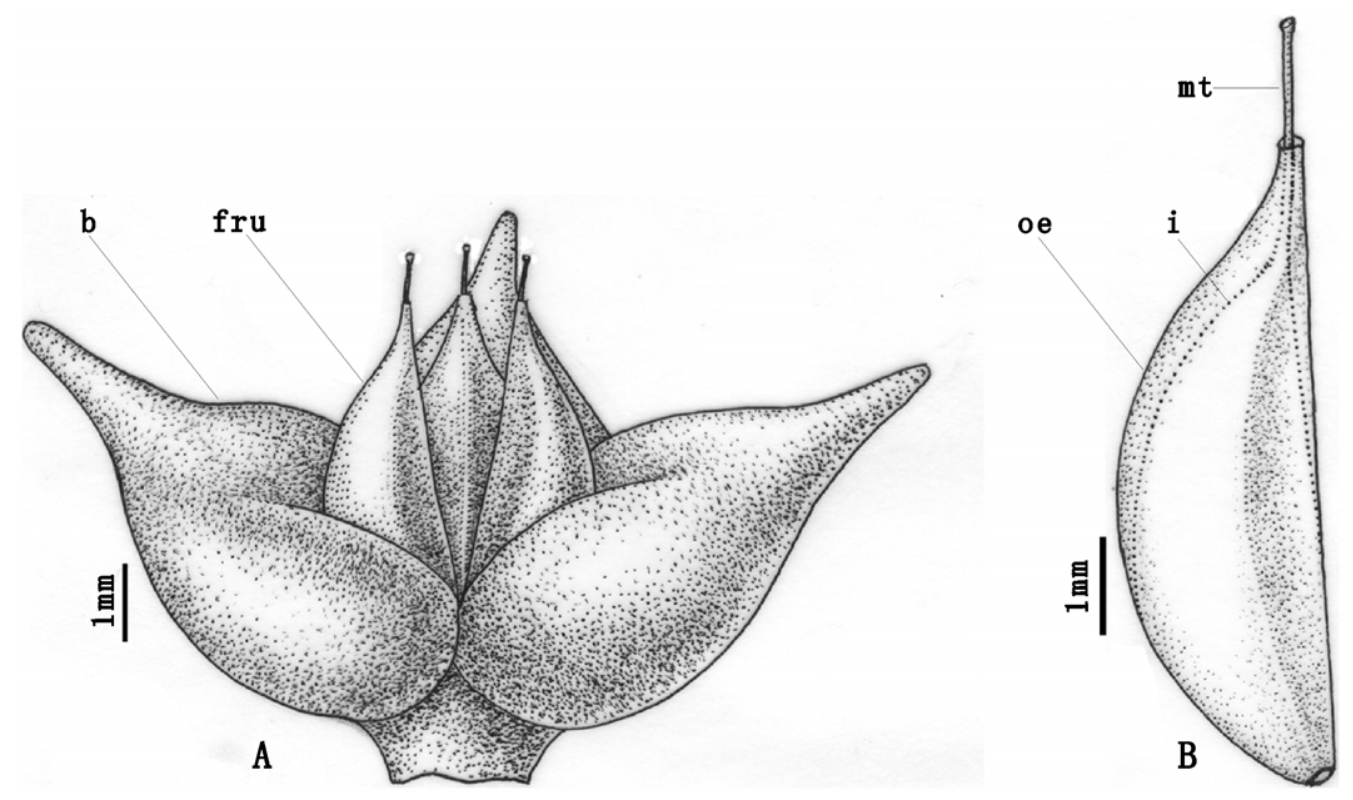

Figure 5. A schematic reconstruction of fossil Ephedra carnosa female cone and its seed. A. The triovulate cone of $E$. carnosa, bearing three spreading, fleshy bracts (b) and three female reproductive units (fru). B. The seed, showing the outer envelope (oe), inner integument (i) and a micropylar tube $(\mathrm{mt})$.

doi:10.1371/journal.pone.0053652.g005

beginning of fleshy cones in response to animal (probably reptiles and birds) dispersal, and subsequent thickened outer envelope would have been evolved into the modern form; the other hypothesis is that the fossil species could not adapt to animal dispersal and become extinct because of its thin envelope. Hence it is an evolutionary blind alley of Ephedra.

\section{Ecological implications for the fleshy bracts}

Three kinds of agents are known for the dispersal of Ephedra. The dry-winged, membranous bracts type of mature female cones is dispersed by wind while the coriaceous bract type is distributed by seed-catching rodents, and the fleshy bract type is dispersed by frugivorous birds [14,15]. During Jurassic and Cretaceous, vertebrate-mediated (e.g., early mammals and early birds) seed dispersal interactions may have been important drivers of seed cone evolution in such conifers as Podocarpaceae Endl. and Taxaceae S. F. Grey, resulting in shifts of female cones from the lax open cones typical of Paleozoic and Triassic conifers to the more compact and reduced seed cones that are associated with fleshiness [53]. Remarkably, a seed-eating bird fossil has been discovered from the Yixian Formation of western Liaoning

\section{References}

1. Gifford EM, Foster AS (1989). Comparative morphology of vascular plants, third edition. New York: WH Freeman. 626p.

2. Stevenson DW (1993) Ephedraceae. In: Flora of North America Editorial Committee, edtors. Flora of North America North of Mexico, vol. 2. New York: Oxford University Press. pp. 428-434

3. Price RA (1996) Systematics of Gnetales: a review of morphological and molecular evidence. Int J Plant Sci 157: S40-S49.

4. Fu LG, Yu YF, Harald R (1999) Ephedraceae. In: Wu ZY, Raven PH, editors. Flora of China, volume 4. Beijing: Science Press \& St. Louis: Missouri Botanical Garden. pp. 97-101.

5. Caveney S, Charlet DA, Freitag H, Maier-Stolte M, Starratt AN (2001) New observations on the secondary chemistry of world Ephedra (Ephedraceae). Am J Bot 88: 1199-1208.

6. Yang Y (2002) Systematics and evolution of Ephedra L. (Ephedraceae) from China. Unpublished Ph. D. dissertation. Beijing: Institute of Botany, Chinese Academy of Sciences. 231p.
[39,41]. Probably this is also the case in Ephedra, so fleshiness as an effective vertebrate-mediated seed dispersal mechanism may have accounted for the wide distribution of ephedroid plants in southern Europe, northeastern Asia, eastern North America, and South America during the Early Cretaceous.

\section{Acknowledgments}

We greatly thank the academic editor Dr. Lee Newsom, the Pennsylvania State University, Dr. Jason Hilton, University of Birmingham, and an anonymous reviewer for their invaluable suggestions; D. Z. Fu, Institute of Botany, CAS for his helpful discussions; H. Wang, Beipiao City for his help in fossil collecting; Y. B. Sun, Institute of Botany, CAS for his excellent line-drawing; S. Shen, Institute of Botany, CAS for her logistics help; J. Y. Guo, the Claremont Colleges, C. H. Xiong, Peking University, and G. L. Shi, Nanjing Institute of Geology and Palaeontology, CAS for providing useful literature.

\section{Author Contributions}

Conceived and designed the experiments: YY QW. Performed the experiments: YY QW. Analyzed the data: YY QW. Contributed reagents/materials/analysis tools: YY QW. Wrote the paper: YY QW.

7. Anderson JM, Anderson HM, Cleal CJ (2007) Brief history of the gymnosperms: classification, biodiversity, phytogeography and ecology. Strelitzia 20. Pretoria: South African National Biodiversity Institute. 280p.

8. Stapf O (1889) Die Arten der Gattung Ephedra. Denkschr Kaiserl Akad Wiss, Wien Math-Naturwiss Kl 56: 1-112.

9. Ickert-Bond SM, Wojciechowski MF (2004) Phylogenetic relationships in Ephedra (Gnetales): evidence from nuclear and chloroplast DNA sequence data. Syst Bot 29: 834-849.

10. Huang JL, Giannasi DE, Price RA (2005) Phylogenetic relationships in Ephedra (Ephedraceae) inferred from chloroplast and nuclear DNA sequences. Mol Phylogen Evol 35: 48-59.

11. Ickert-Bond SM, Rydin C, Renner SS (2009) A fossil-calibrated relaxed clock for Ephedra indicates an Oligocene age for the divergence of Asian and New World clades and Miocene dispersal into South America. J Syst Evol 47: 444-456.

12. Rydin G, Korall P (2009) Evolutionary relationships in Ephedra (Gnetales), with implications for seed plant phylogeny. Int J Plant Sci 170: 1031-1043. 
13. Ridley HN (1930) The dispersal of plants throughout the world. Ashford: L. Reeve. 744p.

14. Hollander JL, Wall SBV (2009) Dispersal syndromes in North American Ephedra. Int J Plant Sci 170: 323-330.

15. Hollander JL, Wall SBV, Baguley JG (2010) Evolution of seed dispersal in North American Ephedra. Evol Ecol 24: 333-345.

16. Huang JL, Price RA (2003) Estimation of the age of extant Ephedra using chloroplast $r b c \mathrm{~L}$ sequence data. Mol Biol Evol 20: 435-440.

17. Yang Y, Geng BY, Dilcher DL, Chen ZD, Lott TA (2005) Morphology and affinities of an Early Cretaceous fossil-Ephedra archaeorhytidosperma sp. nov. (Ephedraceae - Gnetopsida). Am J Bot 92: 231-241.

18. Rydin C, Pedersen KR, Crane PR, Friis EM (2006) Former diversity of Ephedra (Gnetales): evidence from Early Cretaceous seeds from Portugal and North America. Ann Bot 98: 123-140.

19. Wang X, Zheng SL (2010) Whole fossil plants of Ephedra and their implications on the morphology, ecology and evolution of Ephedraceae (Gnetales). Chin Sci Bull 55: 675-683.

20. Yang Y (2011) The minimum age of Ephedra L. (Ephedraceae): conflicting results between molecular clock and megafossils. Geol Rev 57: 9-15.

21. Rydin G, Wu SQ Friis EM (2006) Liaoxia (Gnetales): ephedroids from the Early Cretaceous Yixian Formation in Liaoning, northeastern China. Pl Syst Evol 262: 239-265.

22. Rydin C, Friis EM (2010) A new Early Cretaceous relative of Gnetales: Siphonospermum simplex gen. et sp. nov. from the Yixian Formation of Northeast China. BMC Evol Biol 10: 183.

23. Guo SX, Wu XW (2000) Ephedrites from Latest Jurassic Yixian Formation in western Liaoning, Northeast China. Acta Palaeontol Sin 39: 81-91.

24. Rydin C, Pedersen KR, Friis EM (2004) On the evolutionary history of Ephedra: Cretaceous fossils and extant molecules. Proc Natl Acad Sci USA 101: 1657116576.

25. Cladera G, Fueyo GMD, de Seoane LV, Archangelsky S (2007) Early Cretaceous riparian vegetation in Patagonia, Argentina. Rev Mus Argent Cienc Nat, N S 9: 49-58.

26. Kunzmann L, Mohr BAR, Bernardes-de-Oliveira MEC (2009) Cearania heterophylla gen. nov. et sp. nov., a fossil gymnosperm with affinities to the Gnetales from the Early Cretaceous of northern Gondwana. Rev Palaeobot Palynol 158: 193-212.

27. Krassilov VA (1982) Early Cretaceous flora of Mongolia. Palaeontogr Abt B 181: 1-43.

28. Krassilov VA (2009) Diversity of Mesozoic gnetophytes and the first angiosperms. Paleontol J 43: 1272-1280.

29. Liu HM, Ferguson DK, Hueber FM, Li CS, Wang YF (2008) Taxonomy and systematics of Ephedrites cheniae and Alloephedra xingxuei (Ephedraceae). Taxon 57: $577-582$.

30. Yang Y (2010) A review on Gnetalean megafossils: problems and perspectives. Taiwania 55: 346-354

31. Yang Y (2007) The nomenclature of fossil Ephedraceae. Taxon 56: 1271-1273.

32. Duan SY (1998) The oldest angiosperm - a tricarpous female reproductive fossil from western Liaoning Province, NE China. Sci China (Ser D) 41: 14-20.

33. Sun G, Zheng SL, Dilcher DL, Wang YD, Mei SW (2001) Early angiosperms and their associated plants from western Liaoning, China. Shanghai: Shanghai Scientific and Technological Education Publishing House. 227p.
34. Swisher CCIII, Wang Y, Wang X, Xu X, Wang Y (1999) Cretaceous age for the feathered dinosaurs of Liaoning, China. Nature 400: 58-61.

35. Barrett PM (2000) Evolutionary consequences of dating the Yixian Formation. Trends Ecol Evol 15: 99-103.

36. Zhou ZH, Barrett PM, Hilton J (2003) An exceptionally preserved Lower Cretaceous ecosystem. Nature 421: 807-814

37. Swisher CCIII, Wang X, Zhou Z, Wang Y, Jin F, et al. (2002) Further support for a Cretaceous age for the feathered dinosaur beds of Liaoning, China: new 40Ar/39Ar dating of the Yixian and Tuchengzi Formations. Chin Sci Bull 47: 135-138.

38. Gradstein FM, Ogg JG, Smith AG, Bleeker W, Lourens LJ (2004) A new Geological Time Scale, with special reference to Precambrian and Neogene. Episodes 27: 83-100.

39. Zhou ZH (2006) Evolutionary radiation of the Jehol Biota: chronological and ecological perspectives. Geol J 41: 377-393.

40. Sun G, Dilcher DL, Zheng SL, Zhou ZK (1998) In search of the first flower: a Jurassic angiosperm, Archaefructus, from Northeast China. Science 282: 1692 1695.

41. Zhou ZH, Zhang FC (2002) A long-tailed, seed-eating bird from the Early Cretaceous of China. Nature 418: 405-409.

42. Yang Y (2001) Ontogeny and metamorphic patterns of female reproductive organs of Ephedra sinica Stapf (Ephedraceae). Acta Bot Sin 43: 1011-1017.

43. Yang Y (2004) Ontogeny of triovulate cones of Ephedra intermedia and origin of the outer envelope of ovules of Ephedraceae. Am J Bot 91: 361-368.

44. Rydin C, Khodabandeh A, Endress PK (2010) The female reproductive unit of Ephedra (Gnetales): comparative morphology and evolutionary perspectives. Bot J Linn Soc 163: 387-430.

45. Pearson HHW (1929). Gnetales. Cambridge: Cambridge University Press. 191p,

46. Kubitzki K (1990) Gnetaceae. In: Kramer KU, Green PS, editors. The families and genera of vascular plants, volume 1. Pteridophytes and gymnosperms. Berlin: Springer-Verlag. pp. 378-391.

47. Yang Y (2005) A new species of Ephedra L. (Ephedraceae) from Sichuan, China with a note on its systematic significance. Bot Bull Acad Sin 46: 363-366.

48. Yang Y (2011) Cuticular diversity of the seed outer envelope in Ephedra (Ephedraceae), with a discussion on its systematic significance. J Trop Subtrop Bot 19: 1-15.

49. Krassilov VA (1986) New floral structure from the Lower Cretaceous of Lake Baikal area. Rev Palaeobot Palynol 47: 9-16.

50. Friis EM, Crane PR, Pedersen KR, Bengtson S, Donoghue PCJ, et al. (2007) Phase-contrast X-ray microtomography links Cretaceous seeds with Gnetales and Bennettitales. Nature 450: 549-553.

51. Friis EM, Pedersen KR, Crane PR (2009) Early Cretaceous mesofossils from Portugal and eastern North America related to the Bennettitales-Erdtmanithecales-Gnetales group. Am J Bot 96: 252-283.

52. Ickert-Bond SM, Rydin C (2011) Micromorphology of the seed envelope of Ephedra L. (Gnetales) and its relevance for the timing of evolutionary events. Int J Plant Sci 172: 36-48.

53. Leslie A (2011) Shifting functional roles and the evolution of conifer pollenproducing and seed-producing cones. Paleobiology 37: 587-602.

54. Friis EM, Crane PR, Pedersen KR (2011) Early flowers and angiosperm evolution. Cambridge: Cambridge University Press. 585p. 